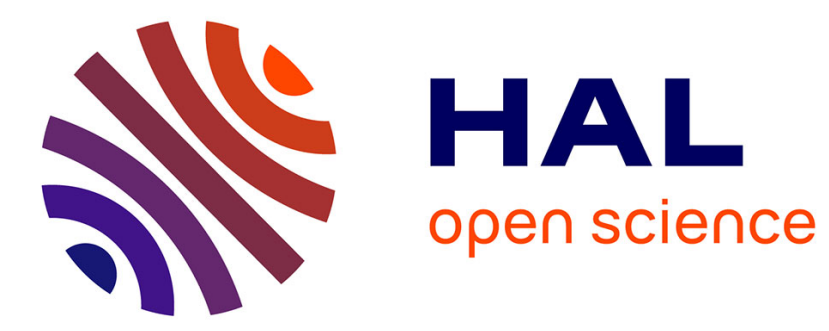

\title{
Extensions of Formal Hodge Structures
}

Nicola Mazzari

\section{- To cite this version:}

Nicola Mazzari. Extensions of Formal Hodge Structures. Communications in Algebra, 2011, 39 (4), pp.1372-1393. 10.1080/00927871003705575 . hal-00772054

\section{HAL Id: hal-00772054 https://hal.science/hal-00772054}

Submitted on 17 Jul 2019

HAL is a multi-disciplinary open access archive for the deposit and dissemination of scientific research documents, whether they are published or not. The documents may come from teaching and research institutions in France or abroad, or from public or private research centers.
L'archive ouverte pluridisciplinaire HAL, est destinée au dépôt et à la diffusion de documents scientifiques de niveau recherche, publiés ou non, émanant des établissements d'enseignement et de recherche français ou étrangers, des laboratoires publics ou privés. 


\title{
Extensions of Formal Hodge Structures
}

\author{
Nicola Mazzari
}

November 11, 2018

\begin{abstract}
We define and study the properties of the category $\mathrm{FHS}_{n}$ of formal Hodge structure of level $\leq n$ following the ideas of L. Barbieri-Viale who discussed the case of level $\leq 1$. As an application we describe the generalized Albanese variety of Esnault, Srinivas and Viehweg via the group $\mathrm{Ext}^{1}$ in $\mathrm{FHS}_{n}$. This formula generalizes the classical one to the case of proper but non necessarily smooth complex varieties.
\end{abstract}

\section{Introduction}

The aim of this work is to develop the program proposed by S. Bloch, L. Barbieri-Viale and V. Srinivas ([BS02],[BV07]) of generalizing Deligne mixed Hodge structures providing a new cohomology theory for complex algebraic varieties. In other words to construct and study cohomological invariants of (proper) algebraic schemes over $\mathbb{C}$ which are finer than the associated mixed Hodge structures in the case of singular spaces. For any natural number $n>0$ (the level) we construct an abelian category, $\mathrm{FHS}_{n}$, and a family of functors

$$
\mathrm{H}_{\sharp}^{n, k}:(\mathrm{Sch} / \mathbb{C})^{\circ} \rightarrow \mathrm{FHS}_{n} \quad 1 \leq k \leq n
$$

such that

1. The category $\mathrm{MHS}_{n}$ of mixed Hodge structure of level $\leq n$ is a full sub-category of $\mathrm{FHS}_{n}$.

2. There is a forgetful functor $f: \mathrm{FHS}_{n} \rightarrow \mathrm{MHS}_{n}$ s.t. $f\left(\mathrm{H}_{\sharp}^{n, k}(X)\right)=$ $H^{n}(X)$ (functorially in $X$ ) is the usual mixed Hodge structure on the Betti cohomology of $X$, i.e. $\mathrm{H}^{n}(X):=\mathrm{H}^{n}\left(X_{\text {an }}, \mathbb{Z}\right)$.

Roughly speaking the sharp cohomology objects $\mathrm{H}_{\sharp}^{n, k}(X)$ consist of the singular cohomology groups $\mathrm{H}^{n}\left(X_{\mathrm{an}}, \mathbb{Z}\right)$, with their mixed Hodge structure, plus some extra structure. We remark that $\mathrm{H}_{\sharp}^{n, k}(X)$ is completely determined by the mixed Hodge structure on $\mathrm{H}^{n}(X)$ when $X$ is proper and smooth; the extra structure shows up only when $X$ is not proper or singular. 
The motivating example is the following. Let $X$ be a proper algebraic scheme over $\mathbb{C}$. Denote $\mathrm{H}^{i}(X):=\mathrm{H}^{i}\left(X_{\text {an }}, \mathbb{Z}\right), \mathrm{H}^{i}(X)_{\mathbb{C}}:=\mathrm{H}^{i}(X) \otimes \mathbb{C}$ and let $\mathrm{H}_{\mathrm{dR}}^{i, j}(X):=\mathrm{H}^{i}\left(X_{\mathrm{an}}, \Omega^{<j}\right)$ be the truncated analytic De Rham cohomology of $X$. Then there is a commutative diagram

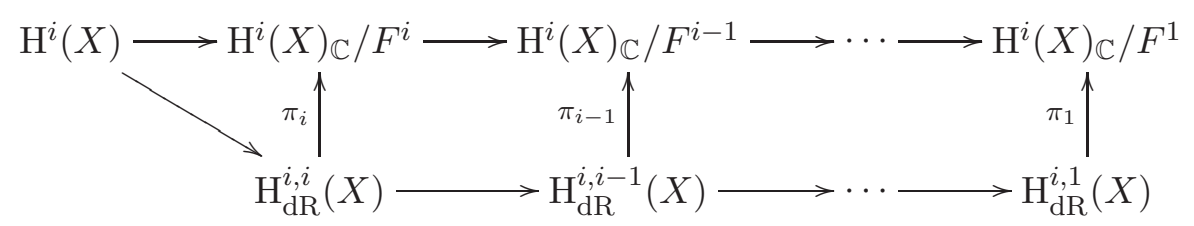

where the $\mathbb{C}$-linear maps $\pi_{j}$ are surjective. This diagram is the formal Hodge structure $\mathrm{H}_{\sharp}^{i, i}(X)$ (or simply $\mathrm{H}_{\sharp}^{i}(X)$ ).

Note that this definition is compatible with the theory of formal Hodge structures of level $\leq 1$ developed by L. Barbieri-Viale (See [BV07]). He defined $\mathrm{H}_{\sharp}^{1}(X)$ as the generalized Hodge realization of $\operatorname{Pic}^{0}(X)$, i.e. $\mathrm{H}_{\sharp}^{1}(X):=$ $T_{\oint}\left(\operatorname{Pic}^{0}(X)\right)$ which is explicitly represented by the diagram

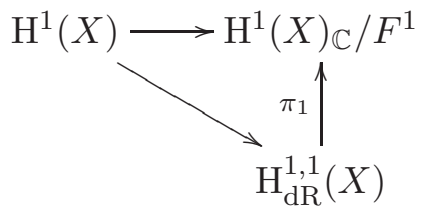

As an application of this theory we can express the Albanese variety of Esnault, Srinivas and Viehweg ([ESV99]) using ext-groups. Precisely let $X$ be a proper, irreducible, algebraic scheme over $\mathbb{C}$. Let $d=\operatorname{dim} X$ and denote by $\mathrm{H}_{\sharp}^{2 d-1, d}(X)$ the formal Hodge structure represented by the following diagram

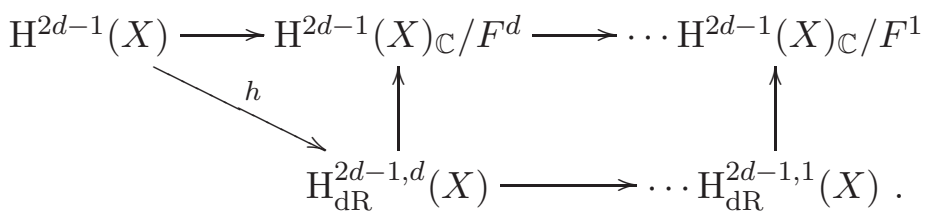

Then there is an isomorphism of complex Lie groups

$$
\operatorname{ESV}(X)_{\mathrm{an}} \cong \operatorname{Ext}_{\mathrm{FHS}_{d}}^{1}\left(\mathbb{Z}(-d), \mathrm{H}_{\sharp}^{2 d-1, d}(X)\right)
$$

where $\operatorname{ESV}(X)$ is the generalized Albanese of [ESV99]. Note that this formula generalizes the classical one

$$
\operatorname{Alb}(X)_{\mathrm{an}} \cong \operatorname{Ext}_{\mathrm{MHS}}^{1}\left(\mathbb{Z}(-d), \mathrm{H}^{2 d-1}(X)\right)
$$

which follows from the work of Carlson (See [Car87]). 


\section{Acknowledgments}

The author would like to thank L. Barbieri-Viale for pointing his attention to this subject and for helpful discussions. The author also thanks A. Bertapelle for many useful comments and suggestions.

\section{Contents}

1 Formal Hodge Structures 3

1.1 Sub-categories of $\mathrm{FHS}_{n} \ldots \ldots \ldots \ldots$

1.2 Adjunctions . . . . . . . . . . . . . . . . . . . 9

1.3 Different levels . . . . . . . . . . . . . . . . . . 9

2 Extensions in $\mathrm{FHS}_{n} \quad 13$

2.1 Basic facts . . . . . . . . . . . . . . . . . . 13

2.2 Formal Carlson theory . . . . . . . . . . . . . 16

3 Sharp Cohomology 17

3.1 The generalized Albanese of Esnault-Srinivas-Viehweg . . . . 20

3.2 The generalized Albanese of Faltings and Wüstholz . . . . . . 21

References

\section{Formal Hodge Structures}

We simply call a formal group a commutative group of the form $H=H^{o} \times H_{\text {et }}$ where $H_{\text {et }}$ is a finitely generated abelian group and $H^{o}$ is a finite dimensional $\mathbb{C}$-vector space. We denote by FrmGrp the category with objects formal groups and morphisms $f=\left(f^{o}, f_{\mathrm{et}}\right): H \rightarrow H^{\prime}$, where $f^{o}: H^{o} \rightarrow H^{\prime o}$ is $\mathbb{C}$-linear and $f_{\text {et }}: H_{\text {et }} \rightarrow H_{\text {et }}^{\prime}$ is $\mathbb{Z}$-linear.

We denote the category of mixed Hodge structures of level $\leq l$ (i.e. of type $\{(n, m) \mid 0 \leq n, m \leq l\})$ by $\mathrm{MHS}_{l}=\mathrm{MHS}_{l}(0)$, for $l \geq 0$. Also we define the category $\mathrm{MHS}_{l}(n)$ to be the full sub-category of MHS whose objects are $H_{\text {et }} \in$ MHS such that $H_{\text {et }} \otimes \mathbb{Z}(-n)$ is in $\operatorname{MHS}_{l}(0)$.

Let $\mathrm{Vec}=\mathrm{Vec}_{1}$ be the category of finite dimensional complex vector spaces and $n>0$ be an integer. We define the category $\mathrm{Vec}_{n}$, as follows. The objects are diagrams of $n-1$ composable arrows of Vec denoted by

$$
V: V_{n} \stackrel{v_{n}}{\longrightarrow} V_{n-1} \stackrel{v_{n-1}}{\longrightarrow} V_{n-2} \rightarrow \cdots \rightarrow V_{1} .
$$

Let $V, V^{\prime} \in \mathrm{Vec}_{n}$, a morphism $f: V \rightarrow V^{\prime}$ is a family $f_{i}: V_{i} \rightarrow V_{i}^{\prime}$ of $\mathbb{C}$-linear maps such that 
is commutative for all $1 \leq i \leq n$.

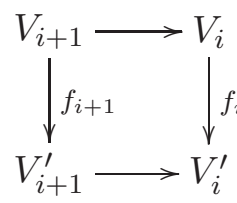

Definition 1.1 (level =0). We define the category of formal Hodge structures of level 0 (twisted by $k$ ), $\mathrm{FHS}_{0}(k)$ as follows: the objects are formal groups $H$ such that $H_{\text {et }}$ is a pure Hodge structure of type $(-k,-k)$; morphism are maps of formal groups.

Equivalently $\mathrm{FHS}_{0}(k)$ is the product category $\mathrm{MHS}_{0}(k) \times \mathrm{Vec}$.

Definition 1.2 (level $\leq n$ ). Fix $n>0$ an integer. We define a formal Hodge structure of level $\leq n$ (or a $n$-formal Hodge structure) to be the data of

i) A formal group $H$ (over $\mathbb{C}$ ) carrying a mixed Hodge structure on the étale component, $\left(H_{\mathrm{et}}, F, W\right)$, of level $\leq n$. Hence we get $F^{n+1} H_{\mathbb{C}}=0$ and $F^{0} H_{\mathbb{C}}=H_{\mathbb{C}}$, where $H_{\mathbb{C}}:=H_{\text {et }} \otimes \mathbb{C}$.

ii) A family of fin. gen. $\mathbb{C}$-vector spaces $V_{i}$, for $1 \leq i \leq n$.

iii) A commutative diagram of abelian groups

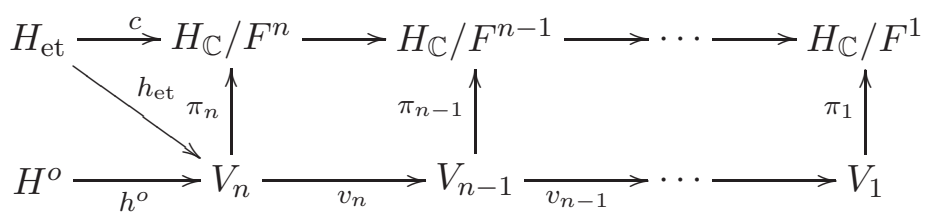

such that $\pi_{i}, h^{o}$ are $\mathbb{C}$-linear maps.

We denote this object by $(H, V)$ or $(H, V, h, \pi)$. Note that $V=\left\{V_{n} \rightarrow \cdots \rightarrow\right.$ $\left.V_{1}\right\}$ can be viewed as an object of $\mathrm{Vec}_{n}$.

The map $h=\left(h_{\mathrm{et}}, h^{o}\right): H \rightarrow V_{n}$ is called augmentation of the given formal Hodge structure.

A morphism of $n$-formal Hodge structures is a pair $(f, \phi)$ such that: $f: H \rightarrow$ $H^{\prime}$ is a morphism of formal groups; $f$ induces a morphism of mixed Hodge structures $f_{\text {et }} ; \phi_{i}: V_{i} \rightarrow V_{i}^{\prime}$ is a family of $\mathbb{C}$-linear maps; $\phi: V \rightarrow V^{\prime}$ is a morphism in $\operatorname{Vec}_{n} ;(f, \phi)$ are compatible with the above structure, i.e. such that the following diagram commutes

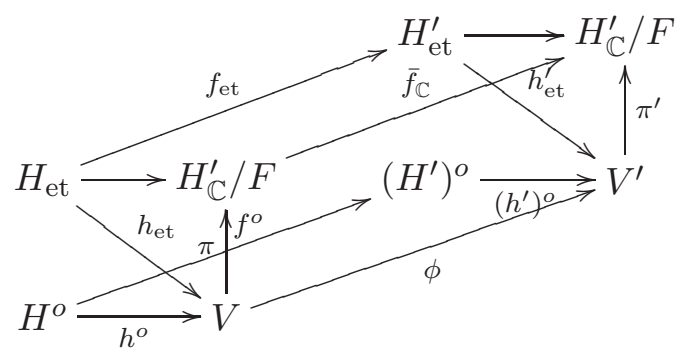

We denote this category by $\mathrm{FHS}_{n}=\mathrm{FHS}_{n}(0)$. 
Remark 1.3. Note that the commutativity of the diagram (iii) of the above definitions implies that the maps $\pi_{i}$ are surjective. In fact after tensor by $\mathbb{C}$ we get that the composition $\pi_{n} \circ h_{\mathbb{C}}$ is the canonical projection $H_{\mathbb{C}} \rightarrow H_{\mathbb{C}} / F^{n}$ : hence $\pi_{n}$ is surjective. Similarly we obtain the surjectivity of $\pi_{i}$ for all $i$.

Example 1.4 (Sharp cohomology of a curve). Let $U=X \backslash D$ be a complex projective curve minus a finite number of points. Then we get the following commutative diagram

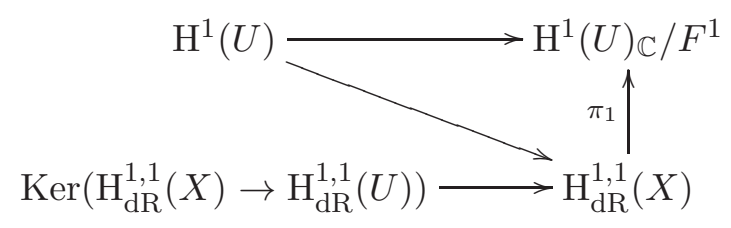

representing a formal Hodge structure of level $\leq 1$.

Remark 1.5 (Twisted fhs). In a similar way one can define the category $\mathrm{FHS}_{n}(k)$ whose object are represented by diagrams

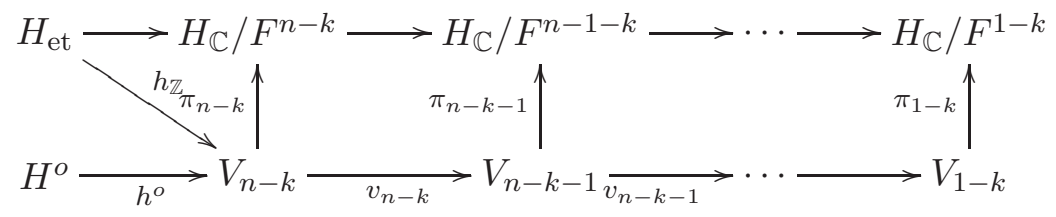

where $H_{\text {et }}$ is an object of $\mathrm{MHS}_{n}(k)$.

Hence the Tate twist $H_{\text {et }} \mapsto H_{\text {et }} \otimes \mathbb{Z}(k)$ induces an equivalence of categories

$$
\mathrm{FHS}_{n}(0) \rightarrow \mathrm{FHS}_{n}(k) \quad(H, V) \mapsto(H(k), V(k))
$$

where $H(k)=H_{\text {et }} \otimes \mathbb{Z}(k) \times H^{o}$ and $V(k)$ is obtained by $V$ shifting the degrees, i.e. $V(k)_{i}=V_{i+k}$, for $1-k \leq i \leq n-k$.

Example 1.6 (Level $\leq 1$ ). According to the above definition a 1-formal Hodge structure twisted by 1 is represented by a diagram

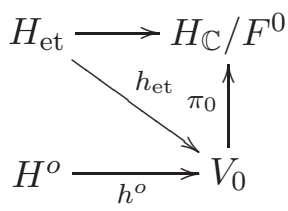

where is $\left(H_{\mathrm{et}}, F, W\right)$ be a mixed Hodge structure of level $\leq 1$ (twisted by $\mathbb{Z}(1)$ ), i.e. of type $[-1,0] \times[-1,0] \subset \mathbb{Z}^{2}$ (recall that this implies $F^{1} H_{\mathbb{C}}=0$ and $F^{-1} H_{\mathbb{C}}=H_{\mathbb{C}}$ ). If we further assume that $H_{\text {et }}$ carries a mixed Hodge structure such that $\operatorname{gr}_{-1}^{W} H_{\text {et }}$ is polarized we get the category studied in [BV07]. 
Proposition 1.7 (Properties of FHS). i) The category $\mathrm{FHS}_{n}$ is an abelian category.

ii) The forgetful functor $(H, V) \mapsto H$ (resp. $(H, V) \mapsto V)$ is an exact functor with values in the category of formal groups (resp. the category $\mathrm{Vec}_{n}$ ).

iii) There exists a full and thick embedding $\mathrm{MHS}_{l}(0) \rightarrow \mathrm{FHS}_{l}(0)$ induced by $\left(H_{\mathrm{et}}, F, W\right) \mapsto\left(H_{\mathrm{et}}, V_{i}=H_{\mathbb{C}} / F^{i}\right)$.

iv) There exists a full and thick embedding $\mathrm{Vec}_{l}(0) \rightarrow \mathrm{FHS}_{l}(0)$ induced by $V \mapsto(0, V)$.

Proof. i) It follows from the fact that we can compute kernels, co-kernels and direct sum component-wise.

ii) It follows by (i).

iii) Let $(f, \phi):\left(H_{\text {et }}, H_{\mathbb{C}} / F\right) \rightarrow\left(H_{\text {et }}^{\prime}, H_{\mathbb{C}}^{\prime} / F\right)$ be a morphism in $\mathrm{FHS}_{\mathrm{n}}$. Then by definition for any $1 \leq i \leq n$ there is a commutative diagram

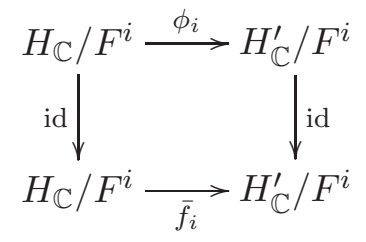

where $\bar{f}_{i}\left(x+F^{i} H_{\mathbb{C}}\right)=f(x)+F^{i} H_{\mathbb{C}}^{\prime}$ is the map induce by $f$ : it is well defined because the morphisms of mixed Hodge structures are strictly compatible w.r.t. the Hodge filtration. Hence $\phi$ is completely determined by $f$.

iv) It is a direct consequence of the definition of $\mathrm{FHS}_{n}$.

Lemma 1.8. Fix $n \in \mathbb{Z}$. The following functor

$$
\mathrm{MHS} \rightarrow \mathrm{Vec}, \quad\left(H_{\mathrm{et}}, W, F\right) \mapsto H_{\mathbb{C}} / F^{n}
$$

is an exact functor.

Proof. This follows from [Del71, §1.2.10].

\subsection{Sub-categories of $\mathrm{FHS}_{n}$}

Let $(H, V)$ be a formal Hodge structure of level $\leq n$. It can be visualized as a diagram

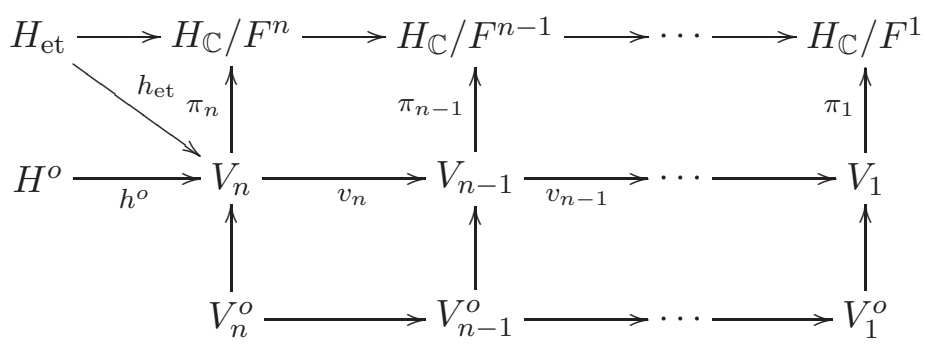


where $V_{i}^{o}:=\operatorname{Ker}\left(\pi_{i}: V_{i} \rightarrow H_{\mathbb{C}} / F^{i}\right)$. We can consider the following $n$-formal Hodge structures

1. $(H, V)_{\mathrm{et}}:=\left(H_{\mathrm{et}}, V / V^{o}\right)$, called the étale part of $(H, V)$.

2. $(H, V)_{\times}:=\left(H, V / V^{o}\right)$, where the augmentation $H \rightarrow H_{\mathbb{C}} / F^{n}=$ $V_{n} / V_{n}^{o}$ is the composite $\pi_{n} \circ h$.

We say that $(H, V)$ is étale (resp. connected) if $(H, V)=(H, V)_{\text {et }}$ (resp. $\left.(H, V)_{\text {et }}=0\right)$. Also we say that $(H, V)$ is special if $h^{o}: H^{o} \rightarrow V_{n}$ factors through $V_{n}^{o}$. We will denote by $\mathrm{FHS}_{n \text {,et }}$ (resp. $\mathrm{FHS}_{n}^{o}, \mathrm{FHS}_{n}^{s}$ ) the full subcategory of $\mathrm{FHS}_{n}$ whose objects are étale (resp. connected, special). Note that by construction the category of étale formal Hodge structure $\mathrm{FHS}_{n \text {,et }}$ is equivalent to $\mathrm{MHS}_{n}$, by abuse of notation we will identify these two categories.

Proposition 1.9 (Canonical Decomposition). i) Let $(H, V) \in \mathrm{FHS}_{n}(n>$ $0)$, then there are two canonical exact sequences

$0 \rightarrow\left(0, V^{o}\right) \rightarrow(H, V) \rightarrow(H, V)_{\times} \rightarrow 0 \quad ; 0 \rightarrow(H, V)_{\mathrm{et}} \rightarrow(H, V)_{\times} \rightarrow\left(H^{o}, 0\right) \rightarrow 0$

ii) The augmentation $h^{o}: H^{o} \rightarrow V_{n}$ factors trough $V_{n}^{o} \Longleftrightarrow$ there is a canonical exact sequence

$$
0 \rightarrow(H, V)^{o} \rightarrow(H, V) \rightarrow(H, V)_{\mathrm{et}} \rightarrow 0
$$

where $(H, V)^{o}:=\left(H^{o}, V^{o}\right)$.

Proof. i) Let $(0, \theta):\left(0, V^{o}\right) \rightarrow(H, V)$ be the canonical inclusion. By 1.7 $\operatorname{Coker}(0, \theta)$ can be calculated in the product category $\operatorname{FrmGrp} \times \operatorname{Vec}_{n}$, i.e. $\operatorname{Coker}(0, \theta)=\operatorname{Coker} 0 \times \operatorname{Coker} \theta=H \times V / V^{o}$ and the augmentation $H \rightarrow$ $H_{\mathbb{C}} / F^{n}$ is the composition $H \stackrel{h}{\rightarrow} V_{n} \stackrel{\pi_{n}}{\rightarrow} H_{\mathbb{C}} / F^{n}$.

For the second exact sequence consider the natural projection $p^{o}: H \rightarrow$ $H^{o}$. It induces a morphism $\left(p^{o}, 0\right):(H, V)_{\times} \rightarrow\left(H^{o}, 0\right)$. Using the same argument as above we get $\operatorname{Ker}\left(p^{o}, 0\right)=\operatorname{Ker} p^{o} \times \operatorname{Ker} 0=H_{\text {et }} \times V / V^{0}$ as an object of FrmGrp $\times \operatorname{Vec}_{n}$. From this follows the second exact sequence.

ii) By the definition of a morphism of formal Hodge structures (of level $\leq n)$ we get that the canonical map, in the category FrmGrp $\times \operatorname{Vec}_{n},\left(p_{\mathbb{Z}}, \pi\right)$ : $H \times V \rightarrow H_{\text {et }} \times V / V^{o}$ induces a morphism of formal Hodge structures $\Longleftrightarrow$ the following diagram commutes

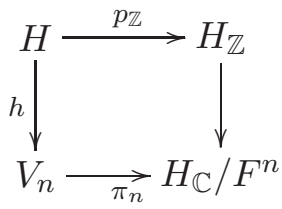

i.e. $\pi_{n} h(x, y)=y \bmod F^{n} H_{\mathbb{C}}$ for all $x \in H^{o}, y \in H_{\text {et }} \Longleftrightarrow h^{o}(x)=0$. 
Remark 1.10. With the above notations consider the map $\left(p^{o}, 0\right): H \times V \rightarrow$ $H^{o} \times 0$. Note that this is a morphism of formal Hodge structure $\Longleftrightarrow$ $V^{0}=0 \Longleftrightarrow(H, V)=(H, V)_{\times}$.

Remark 1.11. For $n=0$ we can also use the same definitions, but the situation is much more easier. In fact a formal structure of level 0 is just a formal group $H$, hence there is a split exact sequence

$$
0 \rightarrow H^{o} \rightarrow H \rightarrow H_{\text {et }} \rightarrow 0
$$

in $\mathrm{FHS}_{0}(0)$.

Corollary 1.12. Let $\mathfrak{K}_{0}\left(\mathrm{FHS}_{\mathrm{n}}\right)$ be the Grothendieck group (see [PSO8, Def. A.4]) associated to the abelian category $\mathrm{FHS}_{n}$. Then

$$
\begin{aligned}
\mathfrak{K}_{0}\left(\mathrm{FHS}_{\mathrm{n}}\right) & =\mathfrak{K}_{0}(\mathrm{Vec}) \times \mathfrak{K}_{0}\left(\mathrm{Vec}_{n}\right) \times \mathfrak{K}_{0}\left(\mathrm{MHS}_{n}\right) \\
& \cong\left\{(f, g) \in \mathbb{Z}[t] \times \mathbb{Z}[u, v] \mid \operatorname{deg}_{t} f, \operatorname{deg}_{u} g, \operatorname{deg}_{v} g \leq n, g(u, v)=g(v, u)\right\}
\end{aligned}
$$

Proof. It follows easily by (i) of 1.9 .

By 1.7 there exists a canonical embedding $\mathrm{MHS}_{n} \subset \mathrm{FHS}_{n}$ (resp. $\mathrm{Vec}_{n} \subset$ $\mathrm{FHS}_{n}$ ). It is easy to check that this embedding gives, in the usual way, a full embedding when passing to the associated homotopy categories, i.e.

$$
K\left(\mathrm{MHS}_{n}\right) \subset K\left(\mathrm{FHS}_{n}\right), \quad \text { resp. } K\left(\mathrm{Vec}_{n}\right) \subset K\left(\mathrm{FHS}_{n}\right) .
$$

With the following lemma we can prove that we have an embedding when passing to the associated derived categories.

Lemma 1.13. Let $\mathrm{A}^{\prime} \subset \mathrm{A}$ be a full embedding of categories. Let $S$ be a multiplicative system in $\mathrm{A}$ and $S^{\prime}$ be its restriction to $\mathrm{A}^{\prime}$. Assume that one of the following conditions

i) For any $s: A^{\prime} \rightarrow A$ (where $A^{\prime} \in \mathrm{A}^{\prime}, A \in \mathrm{A}, s \in S$ ) there exists a morphism $f: A \rightarrow B^{\prime}$ such that $B^{\prime} \in \mathrm{A}^{\prime}$ and $f \circ s \in S$.

ii) The same as (i) with the arrow reversed.

Then the localization $\mathrm{A}_{S^{\prime}}^{\prime}$ is a full sub-category of $\mathrm{A}_{S}$.

Proof. [KS90, 1.6.5].

Proposition 1.14. There is a full embedding of categories $D\left(\mathrm{MHS}_{n}\right) \subset$ $D\left(\mathrm{FHS}_{n}\right)$ (resp. $D\left(\mathrm{Vec}_{n}\right) \subset D\left(\mathrm{FHS}_{n}\right)$ ).

Proof. We will prove only the case involving $\mathrm{MHS}_{n}$, the other one is similar. First note that similarly to (1) there is a full embedding $K\left(\mathrm{FHS}_{n, x}\right) \subset$ $K\left(\mathrm{FHS}_{n}\right)$, where $\mathrm{FHS}_{n, \times}$ is the full sub-category of $\mathrm{FHS}_{n}$ with objects $(H, V)$ such that $(H, V)=(H, V)_{\times}$(See 1.9). Now using $(i)$ of lemma 1.13 and the first exact sequence of 1.9 we get a full embedding $D\left(\mathrm{FHS}_{n, \times}\right) \subset D\left(\mathrm{FHS}_{n}\right)$. Then consider the canonical embedding $\mathrm{MHS}_{n} \subset \mathrm{FHS}_{n, \times}$. Again we get a full embedding of triangulated categories $K\left(\mathrm{MHS}_{n}\right) \subset K\left(\mathrm{FHS}_{n, \times}\right)$. Now using (ii) of lemma 1.13 and the second exact sequence of 1.9 we get a full embedding $D\left(\mathrm{FHS}_{n, \times}\right) \subset D\left(\mathrm{FHS}_{n}\right)$. 


\subsection{Adjunctions}

Proposition 1.15. The following adjunction formulas hold

i) $\operatorname{Hom}_{\mathrm{MHS}}\left(H_{\mathrm{et}}, H_{\mathrm{et}}^{\prime}\right) \cong \operatorname{Hom}_{\mathrm{FHS}_{n}}\left((H, V),\left(H_{\mathrm{et}}^{\prime}, H_{\mathbb{C}}^{\prime} / F\right)\right)$ for all $(H, V) \in$ $\mathrm{FHS}_{n}^{s}$ (i.e. special), $H_{\mathrm{et}}^{\prime} \in \mathrm{MHS}_{n}$.

ii) $\operatorname{Hom}_{\mathrm{FHS}_{n}}\left(\left(H^{o}, V\right),\left(H^{\prime}, V^{\prime}\right)\right) \cong \operatorname{Hom}_{\mathrm{FHS}}\left(\left(H^{o}, V\right),\left(\left(H^{\prime}\right)^{o},\left(V^{\prime}\right)^{o}\right)\right)$ for all $\left(H^{o}, V\right) \in \mathrm{FHS}_{n}^{o}$ (i.e. connected), $\left(H^{\prime}, V^{\prime}\right) \in \mathrm{FHS}_{n}^{s}$.

Proof. The proof is straightforward. Explicitly: i) Let $(H, V) \in \mathrm{FHS}_{n}^{s}, H_{\text {et }}^{\prime} \in$ $\mathrm{MHS}_{n}$. By definition a morphism $(f, \phi) \in \operatorname{Hom}_{\mathrm{FHS}_{n}}\left((H, V),\left(H_{\text {et }}^{\prime}, H_{\mathbb{C}}^{\prime} / F\right)\right)$ is a morphism of formal groups $f: H \rightarrow H^{\prime}$ such that $f_{\text {et }}$ is a morphism of mixed Hodge structures, hence $f=f_{\text {et }}$, and $\phi: V \rightarrow H_{\mathbb{C}}^{\prime} / F$ is subject to the condition $f / F \circ \pi=\phi$. Then the association $(f, \phi) \mapsto f_{\text {et }} \in$ $\operatorname{Hom}_{\mathrm{MHS}}\left(H_{\mathrm{et}}, H_{\mathrm{et}}^{\prime}\right)$ is an isomorphism.

ii) Let $\left(H^{o}, V\right) \in \mathrm{FHS}_{n}^{o},\left(H^{\prime}, V^{\prime}\right) \in \mathrm{FHS}_{n}^{s}$.

A morphism $(f, \phi)$ in $\operatorname{Hom}_{\mathrm{FHS}_{n}}\left(\left(H^{o}, V\right),\left(H^{\prime}, V^{\prime}\right)\right)$ is of the form $f=f^{o}$ : $H^{o} \rightarrow\left(H^{\prime}\right)^{o}, \phi: V \rightarrow V^{\prime}$ must factor through $\left(V^{\prime}\right)^{o}$ because $\pi^{\prime} \circ \phi=$ $\pi \circ f / F=0$.

\subsection{Different levels}

Any mixed Hodge structure of level $\leq n$ (say in $\mathrm{MHS}_{n}(0)$ ) can also be viewed as an object of $\mathrm{MHS}_{m}(0)$ for any $m>n$. This give a sequence of full embeddings

$$
\mathrm{MHS}_{0} \subset \mathrm{MHS}_{1} \subset \cdots \subset \mathrm{MHS}
$$

In this section we want to investigate the analogous situation in the case of formal Hodge structures.

Consider the two functors $\iota, \eta: \mathrm{Vec}_{n} \rightarrow \mathrm{Vec}_{n+1}$ defined as follows

$$
\begin{array}{ll}
\iota(V): & \iota(V)_{n+1}=V_{n} \stackrel{\text { id }}{\rightarrow} \iota(V)_{n}=V_{n} \stackrel{v_{n}}{\rightarrow} \cdots \rightarrow V_{1} \\
\eta(V): & \eta(V)_{n+1}=0 \stackrel{0}{\rightarrow} \iota(V)_{n}=V_{n} \stackrel{v_{n}}{\rightarrow} \cdots \rightarrow V_{1}
\end{array}
$$

Proposition 1.16. The functors $\iota, \eta$ are full and faithful. Moreover the essential image of $\iota$ (resp. $\eta$ ) is a thick sub-category ${ }^{1}$.

Proof. To check that $\iota, \eta$ are embeddings it is straightforward. We prove that the essential image of $\iota$ (resp. $\eta$ ) is closed under extensions only in case $n=2$ just to simplify the notations.

\footnotetext{
${ }^{1}$ By thick we mean a sub-category closed under kernels, co-kernels and extensions
} 
First consider an extension of $\eta V$ by $\eta V^{\prime}$ in $\mathrm{Vec}_{3}$

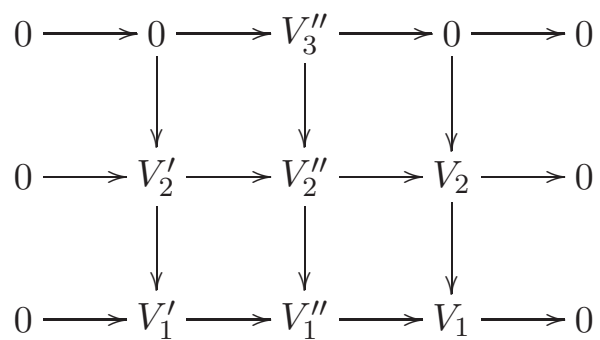

then it follows that $V_{3}^{\prime \prime}=0$.

Now consider an extension of $\iota V$ by $\iota V^{\prime}$ in $\mathrm{Vec}_{3}$

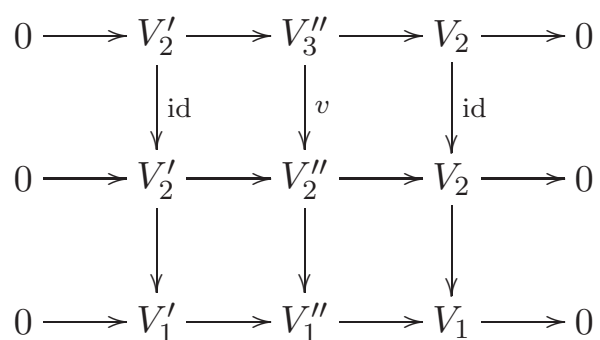

Then $v$ is an isomorphism (by the snake lemma). It follows that $V^{\prime \prime}$ is isomorphic, in $\mathrm{Vec}_{3}$, to an object of $\iota \mathrm{Vec}_{2}$. To check that the essential image of $\iota$ (resp. $\eta$ ) is closed under kernels and cokernels is straightforward.

Remark 1.17. The category of complexes of objects of Vec concentrated in degrees $1, \ldots, n$ is a full sub-category of $\mathrm{Vec}_{n}$. Moreover the embedding induces an equivalence of categories for $n=1$ and 2, but for $n>2$ the embedding is not thick.

Example $1.18\left(\mathrm{FHS}_{1} \subset \mathrm{FHS}_{2}\right)$. The basic construction is the following: let $(H, V)$ be a 1 -fhs, we can associate a 2 -fhs $\left(H^{\prime}, V^{\prime}\right)$ represented by a diagram of the following type

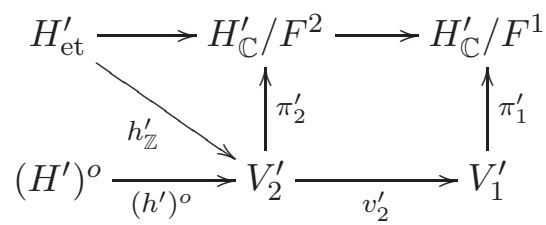

Take $H_{\text {et }}^{\prime}:=H_{\text {et }}$, then $H_{\mathbb{C}}^{\prime} / F^{2}=H_{\mathbb{C}}, H_{\mathbb{C}}^{\prime} / F^{1}=H_{\mathbb{C}} / F^{1}$ and the augmentation $h_{\text {et }}^{\prime}$ is the canonical inclusion; let $V_{1}^{\prime}:=V_{1}, \pi_{1}^{\prime}:=\pi_{1}$ and define $V_{2}^{\prime}, \pi_{2}^{\prime}, v_{2}^{\prime}$ via fiber product

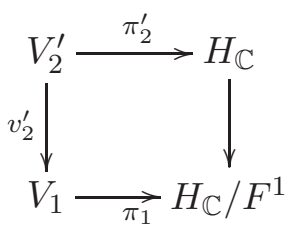


Hence $V_{2}^{\prime}$ fits in the following exact sequences

$$
0 \rightarrow F^{1} H_{\mathbb{C}} \rightarrow V_{2}^{\prime} \rightarrow V_{1} \rightarrow 0 \quad ; \quad 0 \rightarrow V_{1}^{0} \rightarrow V_{2}^{\prime} \rightarrow H_{\mathbb{C}} \rightarrow 0 .
$$

Finally we define $\left(h^{\prime}\right)^{o}:\left(H^{\prime}\right)^{o} \rightarrow V_{2}^{\prime}$ again via fiber product

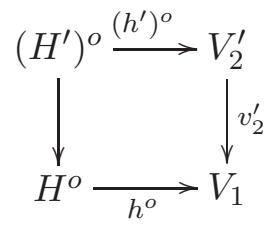

hence we get the following exact sequence

$$
0 \rightarrow F^{1} H_{\mathbb{C}} \rightarrow\left(H^{\prime}\right)^{o} \rightarrow H^{o} \rightarrow 0 .
$$

By induction is easy to extend this construction. We have the following result.

Proposition 1.19. Let $n, k>0$. Then there exists a faithful functor

$$
\iota=\iota_{k}: \mathrm{FHS}_{n} \rightarrow \mathrm{FHS}_{n+k}
$$

Moreover $\iota$ induces an equivalence between $\mathrm{FHS}_{n}$ and the sub-category of $\mathrm{FHS}_{n+k}$ whose objects are $(H, V)$ such that

a) $H_{\mathrm{et}}$ is of level $\leq n$. Hence $F^{n+1} H_{\mathbb{C}}=0$ and $F^{0} H_{\mathbb{C}}=H_{\mathbb{C}}$.

b) $V_{n+i}=V_{n+1}$ for $1 \leq i \leq k$.

c) There exists a commutative diagram with exact rows

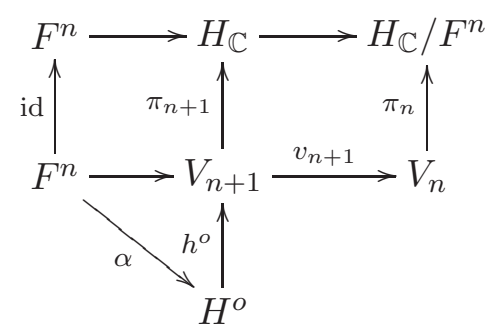

where $\alpha$ is a $\mathbb{C}$-linear map. (c)

And morphisms are those in $\mathrm{FHS}_{n+k}$ compatible w.r.t. the diagram in

Proof. The construction of $\iota_{k}$ is a generalization of that in 1.18. We have $\iota_{k}=\iota_{1} \circ \iota_{k-1}$, hence it is enough to define $\iota_{1}$ which is the same as in $1.18 \mathrm{up}$ to a change of subscripts: $n=1, n+1=2$.

To prove the equivalence we define a quasi-inverse: Let $\left(H^{\prime}, V^{\prime}\right) \in \mathrm{FHS}_{n+1}$ and satisfying $a, b, c$ and $\alpha: F^{n} H_{\mathbb{C}}^{\prime} \rightarrow\left(H^{\prime}\right)^{o}$ as in the proposition.

Define $(H, V) \in \mathrm{FHS}_{n}$ in the following way: $H=H^{\prime} / \alpha\left(F^{n} H_{\mathbb{C}}^{\prime}\right) ; V_{i}=V_{i}^{\prime}$ for all $1 \leq i \leq n ; h: H^{\prime} / \alpha\left(F^{n} H_{\mathbb{C}}^{\prime}\right) \stackrel{\bar{h}^{\prime}}{\longrightarrow} V_{n+1}^{\prime} \stackrel{v_{n+1}^{\prime}}{\longrightarrow} V_{n}^{\prime}=V_{n}$, where $\overline{h^{\prime}}=\left(h_{\text {et }}^{\prime},\left(h^{\prime}\right)^{o}\right.$ $\left.\bmod F^{n}\right)$. 
Proposition 1.20. Let $n, k>0$ and denote by $\iota_{k} \mathrm{FHS}_{n} \subset \mathrm{FHS}_{n+k}$ the essential image of $\mathrm{FHS}_{n}$ (See the previous proposition). Then $\iota_{k} \mathrm{FHS}_{n} \subset \mathrm{FHS}_{n+k}$ is an abelian (not full) sub-category closed under kernels, cokernels and extensions.

Proof. Straightforward.

Remark 1.21. Note that $\iota_{k} \mathrm{FHS}_{n} \subset \mathrm{FHS}_{n+k}$ it is not closed under sub-objects.

Remark 1.22. Let $\mathrm{FHS}_{n}^{\text {prp }}$ be the full sub-category of $\mathrm{FHS}_{n}$ whose objects are formal Hodge structures $(H, V)$ with $H^{o}=0^{2}$. Then $\iota_{k}$ induces a full and faithful functor

$$
\iota=\iota_{k}: \mathrm{FHS}_{n}^{p r p} \rightarrow \mathrm{FHS}_{n+k}^{p r p}
$$

Moreover $\iota_{k} \mathrm{FHS}_{n}^{p r p} \subset \mathrm{FHS}_{n+k}^{p r p}$ is an abelian thick sub-category.

Example 1.23 (Special structures). For special structures it is natural to consider the following construction, similar to $\iota_{k}$ (Compare with 1.18). Let $(H, V)$ be a formal Hodge structures of level $\leq 1$. Define $\tau(H, V)=\left(H, V^{\prime}\right)$ to be the formal Hodge structure of level $\leq 2$ represented by the following diagram

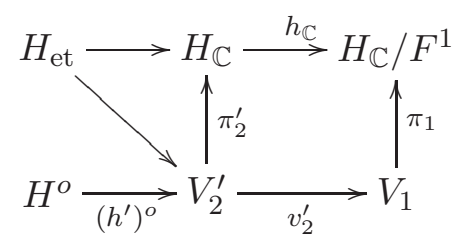

where $V_{2}^{\prime}, v_{2}^{\prime},\left(h^{\prime}\right)^{o}$ are defined via fiber product as follows

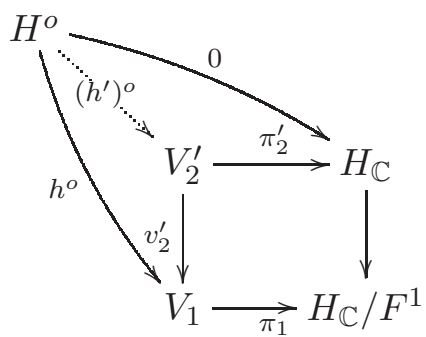

Note that the commutativity of the external square is equivalent to say that $(H, V)$ is special. Hence this construction cannot be used for general formal Hodge structures.

Proposition 1.24. Let $n, k>0$ integers. Then there exists a full and faithful functor

$$
\tau=\tau_{k}: \mathrm{FHS}_{n}^{s} \rightarrow \mathrm{FHS}_{n+k}^{s}
$$

\footnotetext{
${ }^{2}$ The superscript prp stands for proper. In fact the sharp cohomology objects (3.1) of a proper variety have this property.
} 
Moreover the essential image of $\tau_{k}, \tau_{k} \mathrm{FHS}_{n}^{s p c}$, is the full and thick abelian sub-category of $\mathrm{FHS}_{n+k}^{s p c}$ with objects $(H, V)$ such that

a) $H_{\text {et }}$ is of level $\leq n$. Hence $F^{n+1} H_{\mathbb{C}}=0$ and $F^{0} H_{\mathbb{C}}=H_{\mathbb{C}}$.

b) $V_{n+i}=V_{n+1}$ for $1 \leq i \leq k$.

c) $V_{n+1}=H_{\mathbb{C}} \times_{H_{\mathbb{C}} / F^{n}} V_{n}$

Proof. Note that $\tau_{k}=\tau_{1} \circ \tau_{k-1}$, hence is enough to construct $\tau_{1}$. Let $(H, V)$ be a special formal Hodge structure of level $\leq n$, then $\tau_{1}(H, V)$ is defined as in 1.23 up to change the sub-scripts $n=1, n+1=2$.

To prove the equivalence it is enough to construct a quasi-inverse of $\tau_{1}$. Let $\left(H^{\prime}, V^{\prime}\right)$ be a special formal Hodge structure of level $\leq n$ satisfying the conditions $a, b, c$ of the proposition, then define $(H, V) \in \mathrm{FHS}_{n}$ as follows: $H:=H^{\prime} ; V_{i}:=V_{i}^{\prime}$ for all $1 \leq i \leq n ; h=v_{n+1}^{\prime} \circ h^{\prime}$.

Thickness follows directly from the exactness of the functors

$$
(H, V) \mapsto H_{\mathrm{et}}, \quad(H, V) \mapsto V^{o} .
$$

Remark 1.25. The functors $\tau_{k}, \iota_{k}$ agree on the full sub-category of $\mathrm{FHS}_{n}$ formed by $(H, V)$ with $H^{o}=0$.

\section{Extensions in $\mathrm{FHS}_{n}$}

\subsection{Basic facts}

Example 2.1. We describe the ext-groups for $\mathrm{Vec}_{2}$. We have the following isomorphism

$$
\phi: \operatorname{Ext}_{\mathrm{Vec}_{2}}^{1}\left(V, V^{\prime}\right) \stackrel{\sim}{\rightarrow} \operatorname{Hom}_{\mathrm{Vec}}\left(\operatorname{Ker} v, \operatorname{Coker} v^{\prime}\right)
$$

Explicitly $\phi$ associates to any extension class the Ker-Coker boundary map of the snake lemma. To prove it is an isomorphism we argue as follows. The abelian category $\mathrm{Vec}_{2}$ is equivalent to the full sub-category $C^{\prime}$ of $C^{b}(\mathrm{Vec})$ of complexes concentrated in degree 0,1 . Hence the group of classes of extensions is isomorphic. Now let $a: A^{0} \rightarrow A^{1}, b: B^{0} \rightarrow B^{1}$ be two complexes of objects of Vec. Then we have

$$
\operatorname{Ext}_{C^{\prime}}^{1}\left(A^{\bullet}, B^{\bullet}\right)=\operatorname{Ext}_{C^{b}(\mathrm{Vec})}^{1}\left(A^{\bullet}, B^{\bullet}\right)=\operatorname{Hom}_{D^{b}(\mathrm{Vec})}\left(A^{\bullet}, B^{\bullet}[1]\right)
$$

because $C^{\prime}$ is a thick sub-category of $C^{b}(\mathrm{Vec})$.

The category $\mathrm{Vec}$ is of cohomological dimension 0 , then $a: A^{0} \rightarrow A^{1}$ is quasi-isomorphic to Ker $a \stackrel{0}{\rightarrow}$ Coker $a$, similarly for $B^{\bullet}$. It follows that

$$
\begin{aligned}
\operatorname{Hom}_{D^{b}(\mathrm{Vec})}\left(A^{\bullet}, B^{\bullet}[1]\right)= & \operatorname{Hom}_{D^{b}(\operatorname{Vec})}(\operatorname{Ker} a[0] \oplus \operatorname{Coker} a[-1], \operatorname{Ker} b[1] \oplus \operatorname{Coker} b[0]) \\
& =\operatorname{Hom}_{\mathrm{Vec}}(\operatorname{Ker} a, \operatorname{Coker} b) .
\end{aligned}
$$


As a corollary we obtain that $\operatorname{Ext}_{\mathrm{Vec}_{2}}^{1}(V,-)$ is a right exact functor and this is a sufficient condition for the vanishing of $\operatorname{Ext}_{\operatorname{Vec}_{2}}^{i}(,-)$ for $i \leq 2$ (i.e. $V_{e c}$ is a category of cohomological dimension 1.).

Example 2.2. The category $\mathrm{Vec}_{3}$ is of cohomological dimension 1. We argue as in [Maz]. Let $V$ be an object of $\mathrm{Vec}_{3}$, we define the following increasing filtration

$$
W_{-2}=\left\{0 \rightarrow 0 \rightarrow V_{1}\right\} ; W_{-1}=\left\{0 \rightarrow V_{2} \rightarrow V_{1}\right\} ; W_{0}=V
$$

Note that morphisms in $\mathrm{Vec}_{3}$ are compatible w.r.t. this filtration. To prove that $\operatorname{Ext}_{\operatorname{Vec}_{3}}^{2}\left(V, V^{\prime}\right)=0$ it is sufficient to show that $\operatorname{Ext}_{\operatorname{Vec}_{3}}^{2}\left(\operatorname{gr}_{i}^{W} V, \operatorname{gr}_{j}^{W} V^{\prime}\right)=$ 0 for $i, j=-2,-1,0$ (just use the short exact sequences induced by $W$, cf. [Maz, Proof of 2.5]). We prove the case $i=0, j=-2$ leaving to the reader the other cases (which are easier, cf. [Maz, 2.2-2.4]).

Let $\gamma \in \operatorname{Ext}_{\operatorname{Vec}_{3}}^{2}\left(\operatorname{gr}_{0}^{W} V, \operatorname{gr}_{-2}^{W} V^{\prime}\right)=0$, we can represent $\gamma$ by an exact sequence in $\mathrm{Vec}_{3}$ of the following type

$$
0 \rightarrow \mathrm{gr}_{-2}^{W} V^{\prime} \rightarrow A \rightarrow B \rightarrow \operatorname{gr}_{0}^{W} V \rightarrow 0
$$

Let $C=\operatorname{Coker}\left(\operatorname{gr}_{-2}^{W} V^{\prime} \rightarrow A\right)=\operatorname{Ker}\left(B \rightarrow \operatorname{gr}_{0}^{W} V\right)$, then $\gamma=\gamma_{1} \cdot \gamma_{2}$ where $\gamma_{1} \in \operatorname{Ext}_{V_{\text {ec } 3}}^{1}\left(C, \operatorname{gr}_{-2}^{W} V^{\prime}\right), \gamma_{2} \in \operatorname{Ext}_{V_{\text {ec }} 3}^{1}\left(\operatorname{gr}_{0}^{W} V, C\right)$. Arguing as in [Maz, 2.4] we can suppose that $C=\operatorname{gr}_{-1}^{W} C$, hence

$\gamma_{1}=\left[0 \rightarrow \operatorname{gr}_{-2}^{W} V^{\prime} \rightarrow A \rightarrow \operatorname{gr}_{-1}^{W} C \rightarrow 0\right], \gamma_{2}=\left[0 \rightarrow \operatorname{gr}_{-1}^{W} C \rightarrow B \rightarrow \operatorname{gr}_{0}^{W} V \rightarrow 0\right]$

It follows that $A=\left\{0 \rightarrow C_{2} \stackrel{f_{1}}{\longrightarrow} V_{1}^{\prime}\right\}, B=\left\{V_{3} \stackrel{f_{2}}{\longrightarrow} C_{2} \rightarrow 0\right\}$ for some $f_{1}, f_{2}$.

Now consider $D=\left\{V_{3} \stackrel{f_{2}}{\longrightarrow} C_{2} \stackrel{f_{1}}{\longrightarrow} V_{1}^{\prime}\right\} \in \mathrm{Vec}_{3}$, then it is easy to check that

$\gamma_{1}=\left[0 \rightarrow W_{-2} D \rightarrow W_{-1} D \rightarrow \operatorname{gr}_{-1}^{W} D \rightarrow 0\right], \gamma_{2}=\left[0 \rightarrow \operatorname{gr}_{-1} D \rightarrow W_{0} D / W_{-2} D \rightarrow \operatorname{gr}_{0}^{W} D \rightarrow 0\right]$

By [Maz, Lemma 2.1] $\gamma=0$.

Proposition 2.3. Let $H_{\mathrm{et}}$ be a mixed Hodge structure of level $\leq n$ : we consider it as an étale formal Hodge structure. Let $\left(H^{\prime}, V^{\prime}\right)$ be be a formal Hodge structure of level $\leq n$ (for $n>0$ ). Then

i) There is a canonical isomorphism of abelian groups

$$
\operatorname{Ext}_{\mathrm{MHS}}^{1}\left(H_{\mathrm{et}}, H_{\mathrm{et}}^{\prime}\right) \cong \operatorname{Ext}_{\mathrm{FHS}}^{1}\left(H_{\mathrm{et}},\left(H^{\prime}, V^{\prime} / V^{\prime o}\right)\right) .
$$

ii) For any $i \geq 2$ there is a canonical isomorphism

$$
\operatorname{Ext}_{\mathrm{FHS}_{n}}^{i}\left(H_{\mathrm{et}},\left(H^{\prime}, V^{\prime} / V^{\prime o}\right)\right) \cong \operatorname{Ext}_{\mathrm{FHS}}^{i}\left(H_{\mathrm{et}},\left(H^{\prime o}, 0\right)\right) \text {. }
$$

Proof. This follows easily by the computation of the long exact sequence obtained applying $\operatorname{Hom}_{\mathrm{FHS}_{\mathrm{n}}}\left(H_{\mathbb{Z}},-\right)$ to the short exact sequence

$$
0 \rightarrow\left(H^{\prime}, V^{\prime}\right)_{\mathrm{et}} \rightarrow\left(H^{\prime}, V^{\prime}\right)_{\times} \rightarrow\left(H^{\prime o}, 0\right) \rightarrow 0 .
$$


Proposition 2.4. The forgetful functor $(H, V) \mapsto H_{\text {et }}$ induces a surjective morphism of abelian groups

$$
\gamma: \operatorname{Ext}_{\mathrm{FHS}_{n}}^{1}\left((H, V),\left(H^{\prime}, V^{\prime}\right)\right) \rightarrow \operatorname{Ext}_{\mathrm{MHS}}^{1}\left(H_{\mathrm{et}}, H_{\mathrm{et}}^{\prime}\right)
$$

for any $(H, V),\left(H^{\prime}, V^{\prime}\right)$ with $H_{\mathrm{et}}, H_{\mathrm{et}}^{\prime}$ free.

Proof. Recall the extension formula for mixed Hodge structures is (see [PS08, I §3.5])

$$
\operatorname{Ext}_{\mathrm{MHS}}^{1}\left(H_{\mathrm{et}}, H_{\mathrm{et}}^{\prime}\right) \cong \frac{W_{0} \mathcal{H o m}\left(H_{\mathrm{et}}, H_{\mathrm{et}}^{\prime}\right)_{\mathbb{C}}}{F^{0} \cap W_{0}\left(\mathcal{H o m}\left(H_{\mathrm{et}}, H_{\mathrm{et}}^{\prime}\right)_{\mathbb{C}}\right)+W_{0} \mathcal{H o m}\left(H_{\mathrm{et}}, H_{\mathrm{et}}^{\prime}\right)_{\mathbb{Z}}}
$$

more precisely we get that any extension class can be represented by $\tilde{H}_{\text {et }}=$ $\left(H_{\mathrm{et}}^{\prime} \oplus H_{\mathrm{et}}, W, F_{\theta}\right)$ where the weight filtration is the direct sum $W_{i} H_{\mathrm{et}}^{\prime} \oplus W_{i} H_{\mathrm{et}}$ and $F_{\theta}^{i}:=F^{i} H_{\text {et }}^{\prime}+\theta\left(F^{i} H_{\text {et }}\right) \oplus F^{i} H_{\text {et }}$, for some $\theta \in W_{0} \mathcal{H o m}\left(H_{\text {et }}, H_{\text {et }}^{\prime}\right) \mathbb{C}$. It follows that $\tilde{H}_{\mathbb{C}} / F_{\theta}^{i}=H_{\mathbb{C}}^{\prime} / F^{i} \oplus H_{\mathbb{C}} / F^{i}$. Then we can consider the formal Hodge structure of level $\leq n(\tilde{H}, \tilde{V})$ defined as follows: $\tilde{H}_{\text {et }}=\left(H_{\text {et }}^{\prime} \oplus H_{\text {et }}, W, F_{\theta}\right)$ as above; $\tilde{H}^{o}:=\left(H^{\prime}\right)^{o} \oplus H^{o} ; \tilde{V}_{i}:=V_{i}^{\prime} \oplus V_{i}, \tilde{v}_{i}:=\left(v_{i}^{\prime}, v_{i}\right) ; \tilde{h}=\left(h^{\prime}, h\right)$. Then it easy to check that $(\tilde{H}, \tilde{V}) \in \operatorname{Ext}_{\mathrm{FHS}_{n}}^{1}\left(\left(H^{\prime}, V^{\prime}\right),(H, V)\right)$ and $\gamma(\tilde{H}, \tilde{V})=$ $\left(H_{\text {et }}^{\prime} \oplus H_{\text {et }}, W, F_{\theta}\right)$.

Example 2.5 (Infinitesimal deformation). Let $f: \widehat{X} \rightarrow \operatorname{Spec} \mathbb{C}[\epsilon] /\left(\epsilon^{2}\right)$ a smooth and projective morphism. Write $X / \mathbb{C}$ for the smooth and projective variety corresponding to the special fiber, i.e. the fiber product

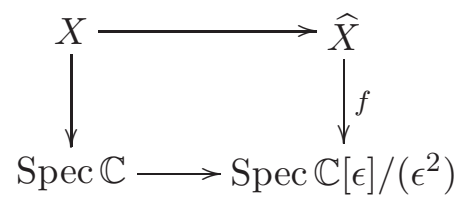

then (see $[\mathrm{BS} 02,2.4])$ for any $i, n$ there is a commutative diagram with exact rows

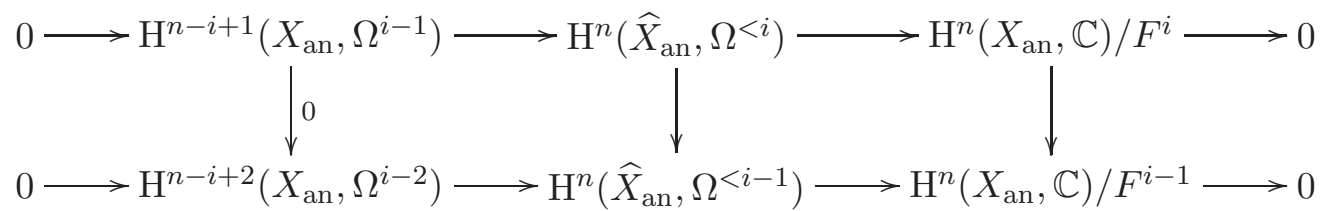

Hence there is an extension of formal Hodge structures of level $\leq n$

$$
0 \rightarrow(0, V) \rightarrow\left(\mathrm{H}^{n}(X), \mathrm{H}_{\mathrm{dR}}^{n, *}(\widehat{X})\right) \rightarrow \mathrm{H}^{n}(X) \rightarrow 0
$$

with $V_{i}=\mathrm{H}^{n-i+1}\left(X_{\mathrm{an}}, \Omega^{i-1}\right)$ and $v_{i}=0$.

Remark 2.6. It is well known that the groups $\operatorname{Ext}^{i}(A, B)$ vanish in category of mixed Hodge structures for any $i>1$. It is natural to ask if the groups $\operatorname{Ext}_{\mathrm{FHS}}^{i}\left((H, V),\left(H^{\prime}, V^{\prime}\right)\right)$ vanish for $i>n$ (up to torsion). In particular Bloch and Srinivas raised a similar question for special formal Hodge structures (cf. [BS02]).

The author answered positively this question for $n=1$ in [Maz]. 


\subsection{Formal Carlson theory}

Proposition 2.7. Let $A, B$ torsion-free mixed Hodge structures. Suppose $B$ pure of weight $2 p$ and $A$ of weights $\leq 2 p-1$. There is a commutative diagram of complex Lie group

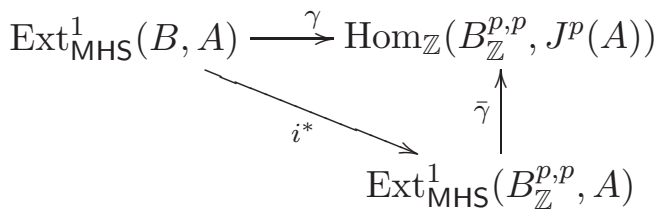

where $\bar{\gamma}$ is an isomorphism; $i^{*}$ is the surjection induced by the inclusion $i: B_{\mathbb{Z}}^{p, p} \rightarrow B$.

Proof. This follows easily from the explicit formula 2. The construction of $\gamma, \bar{\gamma}$ is given in the following remark. Then choosing a basis of $B_{\mathbb{Z}}^{p, p}$ it is easy to check that $\bar{\gamma}$ is an isomorphism.

Remark 2.8. i) Let $\left\{b_{1}, \ldots, b_{n}\right\}$ a $\mathbb{Z}$-basis of $B_{\mathbb{Z}}^{p, p}$, then $\operatorname{Hom}_{\mathbb{Z}}\left(B_{\mathbb{Z}}^{p, p}, J^{p}(A)\right) \cong$ $\oplus_{i=1}^{n} J^{p}(A)$ which is a complex Lie group.

ii) Explicitly $\gamma$ can be constructed as follows. Let $x \in \operatorname{Ext}_{\mathrm{MHS}}^{1}(B, A)$ represented by the extension

$$
0 \rightarrow A \rightarrow H \rightarrow B \rightarrow 0
$$

then apply $\operatorname{Hom}_{\mathrm{MHS}}(\mathbb{Z}(-p),-)$ to the above exact sequence and consider the boundary of the associated long exact sequence

$$
\cdots \rightarrow \operatorname{Hom}_{\mathrm{MHS}}(\mathbb{Z}(-p), B) \stackrel{\partial_{x}}{\longrightarrow} \operatorname{Ext}_{\mathrm{MHS}}^{1}(\mathbb{Z}(-p), A) \rightarrow \cdots
$$

Note that $\partial_{x}$ does not depend on the choice of the representative of $x$; $\operatorname{Hom}_{\mathrm{MHS}}(\mathbb{Z}(-p), B)=B_{\mathbb{Z}}^{p, p} ; J^{p}(A)=\operatorname{Ext}_{\mathrm{MHS}}^{1}(\mathbb{Z}(-p), A)$.

Hence we can define $\gamma(x):=\partial_{x} \in \operatorname{Hom}_{\mathbb{Z}}\left(B_{\mathbb{Z}}^{p, p}, J^{p}(A)\right)$.

iii) If the complex Lie group $J^{p}(A)$ is algebraic then $\operatorname{Hom}_{\mathbb{Z}}\left(B_{\mathbb{Z}}^{p, p}, J^{p}(A)\right)$ can be identified with set of one motives of type

$$
u: B_{\mathbb{Z}}^{p, p} \rightarrow J^{p}(A)
$$

Definition 2.9 (formal-p-Jacobian). Let $(H, V)$ be a formal Hodge structure of level $\leq n$. Assume $H_{\text {et }}$ is a torsion free mixed Hodge structure. For $1 \leq p \leq n$ the $p$-th formal Jacobian of $(H, V)$ is defined as

$$
J_{\sharp}^{p}(H, V):=V_{p} / H_{\text {et }} .
$$

where $H_{\text {et }}$ acts on $V_{p}$ via the augmentation $h$. By construction there is an extension of abelian groups

$$
0 \rightarrow V_{p}^{0} \rightarrow J_{\sharp}^{p}(H, V) \rightarrow J^{p}(H, V) \rightarrow 0
$$

where we define $J^{p}(H, V):=J^{p}\left(H_{\mathrm{et}}\right)=H_{\mathbb{C}} /\left(F^{p}+H_{\mathrm{et}}\right)$. 
Note that that $J_{\sharp}^{p}(H, V)$ is a complex Lie group if the weights of $H_{\text {et }}$ are $\leq 2 p-1$.

Proposition 2.10. There is an extension of abelian groups

$$
0 \rightarrow V_{p}^{o} \rightarrow \operatorname{Ext}_{\mathrm{FHS}_{p}}^{1}(\mathbb{Z}(-p),(H, V)) \rightarrow \operatorname{Ext}_{\mathrm{MHS}}^{1}\left(\mathbb{Z}(-p), H_{\mathrm{et}}\right) \rightarrow 0
$$

for any $(H, V)$ formal Hodge structure of level $\leq p+1$. In particular if $H_{\mathrm{et}}$ has weights $\leq 2 p-1$ there is an extension

$$
0 \rightarrow V_{p}^{o} \rightarrow \operatorname{Ext}_{\mathrm{FHS}_{p}}^{1}(\mathbb{Z}(-p),(H, V)) \rightarrow J^{p}\left(H_{\mathrm{et}}\right) \rightarrow 0 .
$$

Proof. By 2.4 there is a surjective map

$$
\gamma: \operatorname{Ext}_{\mathrm{FHS}_{p}}^{1}(\mathbb{Z}(-p),(H, V)) \rightarrow \operatorname{Ext}_{\mathrm{MHS}}^{1}\left(\mathbb{Z}(-p), H_{\mathrm{et}}\right) .
$$

Recall that $\mathbb{Z}(-p)$ is a mixed Hodge structure and here is considered as a formal Hodge structure of level $\leq p$ represented by the following diagram

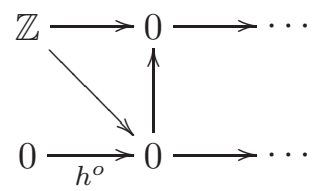

It follows directly from the definition of a morphism of formal Hodge structures that an element of Ker $\gamma$ is a formal Hodge structure of the form $(H \times \mathbb{Z}(-p), H / F)$ represented by

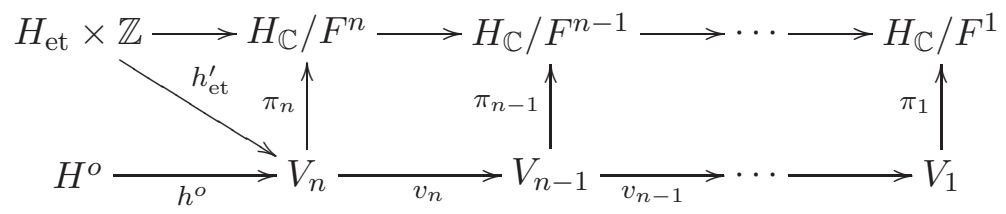

where the augmentation $h_{\mathrm{et}}^{\prime}(x, z)=h_{\mathrm{et}}(x)+\theta(z)$ for some $\theta: \mathbb{Z} \rightarrow V_{p}^{o}$. The map $\theta$ does not depend on the representative of the class of the extension because $V_{p}$ and $\mathbb{Z}(-p)$ are fixed.

Example 2.11. By the previous proposition for $p=1$ we get

$$
0 \rightarrow V_{1}^{o} \rightarrow \operatorname{Ext}_{\mathrm{FHS}_{1}}^{1}(\mathbb{Z}(-1),(H, V)) \rightarrow \operatorname{Ext}_{\mathrm{MHS}}^{1}\left(\mathbb{Z}(-1), H_{\mathrm{et}}\right) \rightarrow 0
$$

\section{Sharp Cohomology}

Definition 3.1. Let $X$ be a proper scheme over $\mathbb{C}, n>0$ and $1 \leq k \leq n$. We define the sharp cohomology object $\mathrm{H}_{\sharp}^{n, k}(X)$ to be the $n$-formal Hodge 
structure represented by the following diagram

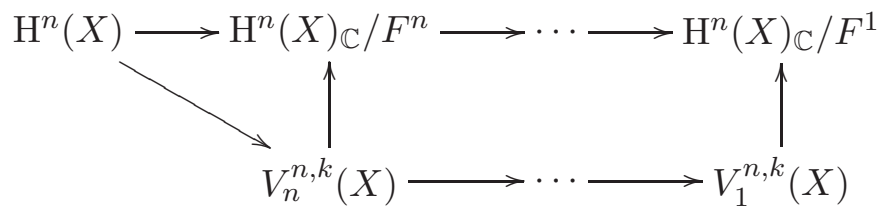

where

$$
V_{i}^{n, k}(X):= \begin{cases}\mathrm{H}_{\mathrm{dR}}^{n, i}(X) & \text { if } 1 \leq i \leq k \\ \mathrm{H}^{n}(X)_{\mathbb{C}} / F^{i} \times_{\mathrm{H}^{n}(X)_{\mathbb{C}} / F^{k}} \mathrm{H}_{\mathrm{dR}}^{n, k}(X) & \text { if } k<i \leq n\end{cases}
$$

In the case $n=k$ we will simply write $\mathrm{H}_{\sharp}^{n}(X)=\mathrm{H}_{\sharp}^{n, n}(X)$. This object is represented explicitly by

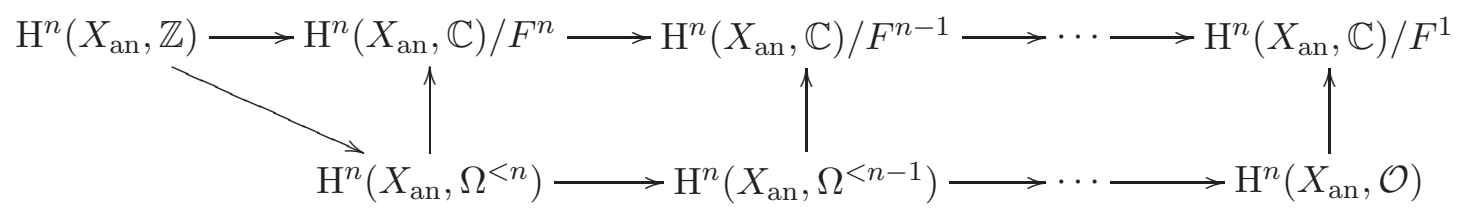

Example 3.2. Let $X$ be a proper scheme of dimension $d$ (over $\mathbb{C}$ ). Then $\mathrm{H}^{2 d-1}(X)$ is a mixed Hodge structure satisfying $F^{d+1}=0$ and the sharp cohomology object $\mathrm{H}_{\sharp}^{2 d-1, d}(X)$ is represented by

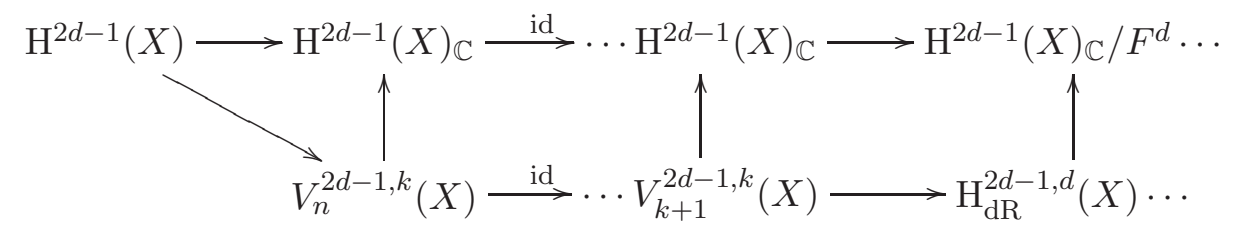

and

$$
F^{d+1} \mathrm{H}^{2 d-1}(X)_{\mathbb{C}} \subset V_{n}^{2 d-1, k}(X)=V_{n-1}^{2 d-1, k}(X)=\cdots=V_{k+1}^{2 d-1, k}(X)
$$

Hence, according to Proposition 1.19, $\mathrm{H}_{\sharp}^{2 d-1, d}(X)$ can be viewed as a formal Hodge structure of level $\leq d$.

Proposition 3.3. For any $n$ and $1 \leq p \leq n$, the association $X \mapsto \mathrm{H}_{\sharp}^{n, p}(X)$ induces a contravariant functor from the category of proper complex algebraic schemes to the category $\mathrm{FHS}_{n}$.

Proof. It is enough to prove the claim for $p=n$. We know that $\mathrm{H}^{n}(X):=$ $\mathrm{H}^{n}\left(X_{\text {an }}, \mathbb{Z}\right)$ along with its mixed Hodge structures is functorial in $X$, so for any $f: X \rightarrow Y$ we have $\mathrm{H}^{n}(f): \mathrm{H}^{n}(Y) \rightarrow \mathrm{H}^{n}(X)$. Also by the theory of Kähler differentials there exist a map of complexes of sheaves over $X$, $\phi_{\bullet}: f^{*} \Omega_{Y}^{\bullet} \rightarrow \Omega_{X}^{\bullet}$, inducing

$$
\alpha: \mathrm{H}^{n}\left(X, f^{*} \Omega_{Y}^{<r}\right) \longrightarrow \mathrm{H}^{n}\left(X, \Omega_{X}^{<r}\right)
$$


Moreover there exists $\beta: \mathrm{H}^{n}\left(Y, \Omega_{Y}^{<r}\right) \rightarrow \mathrm{H}^{n}\left(X, f^{*} \Omega_{Y}^{<r}\right)$. For it is sufficient to construct a map $\beta^{\prime}: \mathrm{H}^{n}\left(Y, \Omega_{Y}^{<r}\right) \rightarrow \mathrm{H}^{n}\left(X, f^{-1} \Omega_{Y}^{<r}\right)$. So let $I^{\bullet}\left(\right.$ resp. $\left.J^{\bullet}\right)$ an injective resolution ${ }^{3}$ of $\Omega_{Y}^{<r}$ (resp. $f^{-1} \Omega_{Y}^{<r}$ ). Using that $f^{-1}$ preserves quasi-isomorphisms, we have the commutative diagram

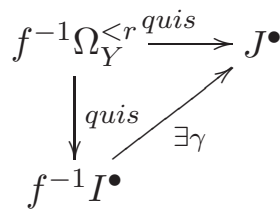

where the existence of $\gamma$ follows from the fact that $J^{\bullet}$ is injective. So we have defined a map $\psi_{r}: \mathrm{H}^{n}\left(Y, \Omega^{<r}\right) \rightarrow \mathrm{H}^{n}\left(X, \Omega^{<r}\right)$.

Now choosing $I_{r}^{\bullet}, J_{r}^{\bullet}$ for any $r$ it's easy to see that the maps $\psi_{r}$ fit in the commutative diagram

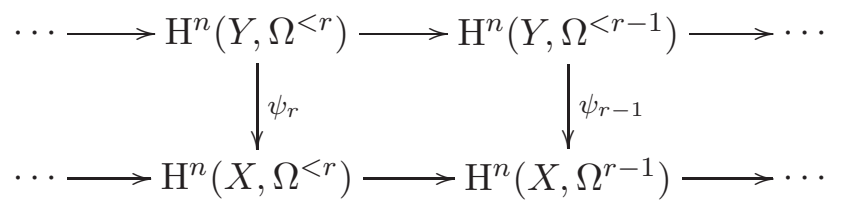

Now it is straightforward to check that $\mathrm{H}_{\sharp}^{n, n}(g \circ f)=\mathrm{H}_{\sharp}^{n, n}(f) \circ \mathrm{H}_{\sharp}^{n, n}(g)$, for any $f: X \rightarrow Y, g: Y \rightarrow Z$.

Example 3.4 (No Künneth). Let $X, Y$ be complete, connected, complex varieties. Then by Künneth formula follows

$$
\mathrm{H}^{1}\left((X \times Y)_{\text {an }}, ?\right)=\mathrm{H}^{1}\left(X_{\text {an }}, ?\right) \oplus \mathrm{H}^{1}\left(Y_{\text {an }}, ?\right) \quad ?=\mathbb{Z}, \mathcal{O}
$$

so that $\mathrm{H}_{\sharp}^{1}(X \times Y)=\mathrm{H}_{\sharp}^{1}(X) \oplus \mathrm{H}_{\sharp}^{1}(Y)$. But as soon as we move in degree 2 there is no hope for a good formula. With the same notation we get

$$
\mathrm{H}^{2}((X \times Y))_{\mathbb{Q}}=\mathrm{H}^{2}(X)_{\mathbb{Q}} \oplus \mathrm{H}^{1}(X)_{\mathbb{Q}} \otimes \mathrm{H}^{1}(Y)_{\mathbb{Q}} \oplus \mathrm{H}^{2}(Y)_{\mathbb{Q}}
$$

which is the usual decomposition of singular cohomology. Let $p: X \times Y \rightarrow X$, $q: X \times Y \rightarrow Y$ the two projections; note that

$$
\mathcal{O}_{X \times Y} \rightarrow \Omega_{X \times Y}^{1}=\sigma^{<2}\left(p^{*}\left(\mathcal{O}_{X} \rightarrow \Omega_{X}^{1}\right) \otimes q^{*}\left(\mathcal{O}_{Y} \rightarrow \Omega_{Y}^{1}\right)\right)
$$

hence there is a canonical map

$$
\mathrm{H}^{2}\left(X \times Y, p^{*}\left(\Omega_{X}^{<2}\right) \otimes q^{*}\left(\Omega_{Y}^{<2}\right)\right)=\oplus_{i=0}^{2} \mathrm{H}_{\mathrm{dR}}^{2-i, 2}(X) \otimes \mathrm{H}_{\mathrm{dR}}^{i, 2}(Y) \rightarrow \mathrm{H}_{\mathrm{dR}}^{2,2}(X \times Y)
$$

which is not necessarily an isomorphism. From this follows that we cannot have a Künneth formula for $\mathrm{H}_{\sharp}^{2,2}(X \times Y)$.

\footnotetext{
${ }^{3}$ By injective resolution of a complex of sheaves $A^{\bullet}$ we mean a quasi isomorphism $A^{\bullet} \rightarrow I^{\bullet}$, where $I^{\bullet}$ is a complex of injective objects.
} 


\subsection{The generalized Albanese of Esnault-Srinivas-Viehweg}

Let $X$ be a proper and irreducible algebraic scheme of dimension $d$ over $\mathbb{C}$. Then there exists an algebraic group, say $\operatorname{ESV}(X)$, such that $\operatorname{ESV}(X)_{\text {an }}=$ $\mathrm{H}^{2 d-1}\left(X, \Omega^{<d}\right) / \mathrm{H}^{2 d-1}\left(X_{\mathrm{an}}, \mathbb{Z}\right)$ and it fits in the following commutative diagram with exact rows

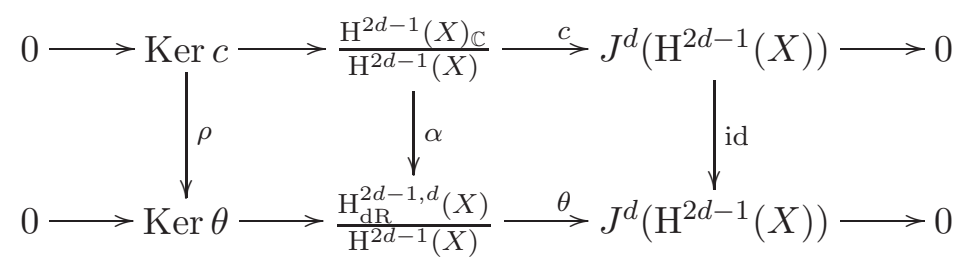

where $\alpha$ is induced by de canonical map of complexes of analytic sheaves $\mathbb{C} \rightarrow \Omega^{<d}$. (See [ESV99, Theorem 1, Lemma 3.1])

Recall that the formal Hodge structure (of level $\leq 2 d-1) \mathrm{H}_{\sharp}^{2 d-1, d}(X)$ can be viewed as a fhs of level $\leq d$ (see 3.2) represented by the following diagram

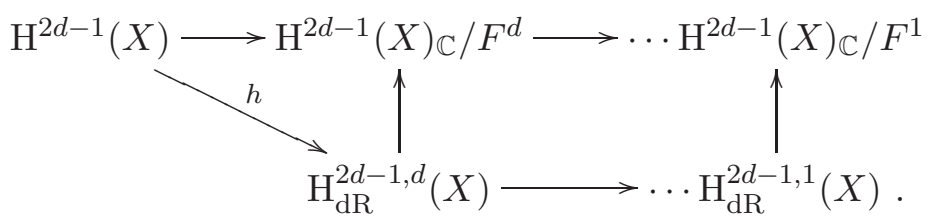

Proposition 3.5. There is an isomorphism of complex connected Lie groups (not only of abelian groups!)

$$
\operatorname{ESV}(X)_{\text {an }} \cong \operatorname{Ext}_{\mathrm{FHS}_{\mathrm{d}}}^{1}\left(\mathbb{Z}(-d), \mathrm{H}_{\sharp}^{2 d-1, d}(X)\right)
$$

where $\mathbb{Z}(-d)$ is the Tate structure of type $(d, d)$ viewed as an étale formal Hodge structure.

Proof. Step 1. By [BV07] there is a canonical isomorphism of Lie groups

$\operatorname{ESV}_{\text {an }}(X) \cong \operatorname{Ext}_{t}^{1} \mathcal{M}_{1}^{\mathrm{a}}([\mathbb{Z} \rightarrow 0],[0 \rightarrow \operatorname{ESV}(X)]) \cong \operatorname{Ext}_{\mathrm{FHS}_{1}(1)}^{1}\left(\mathbb{Z}(0), T_{\oint}(\operatorname{ESV}(X))\right)$

(recall that in [BV07] $\mathrm{FHS}_{1}(1)$ is simply denote by $\mathrm{FHS}_{1} ;{ }^{t} \mathcal{M}_{1}^{\mathrm{a}}$ is the category of generalized 1-motives with torsion) where $T_{\oint}(\operatorname{ESV}(X))$ is the formal Hodge structure represented by

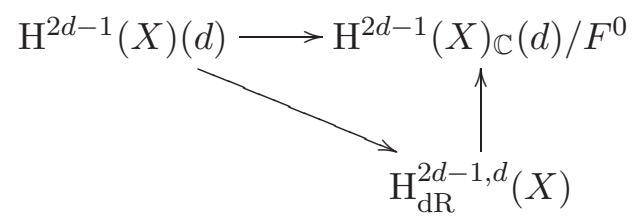


Step 2. Up to a twist by $-d$ we can view $T_{\oint}(\operatorname{ESV}(X))$ as an object of $\mathrm{FHS}_{d}$, say $\left(H_{\mathrm{et}}, V\right)$ with $H_{\mathrm{et}}=\mathrm{H}^{2 d-1}(X), V_{d}=\mathrm{H}_{\mathrm{dR}}^{2 d-1, d}(X), V_{i}=0$ for $1 \leq i<d$. It is easy to check that $\operatorname{Ext}_{\mathrm{FHS}_{1}(1)}^{1}\left(\mathbb{Z}(0), T_{\oint}(\operatorname{ESV}(X))\right)=$ $\operatorname{Ext}_{\mathrm{FHS}_{d}}^{1}\left(\mathbb{Z}(-d),\left(H_{\mathrm{et}}, V\right)\right)$. Then applying $\operatorname{Ext}_{\mathrm{FHS}_{d}}^{1}(\mathbb{Z}(-d),-)$ to the canonical inclusion $\left(H_{\text {et }}, V\right) \subset \mathrm{H}_{\sharp}^{2 d-1, d}(X)$ we get a natural map

$$
\operatorname{Ext}_{\mathrm{FHS}_{1}(1)}^{1}\left(\mathbb{Z}(0), T_{\oint}(\operatorname{ESV}(X))\right) \rightarrow \operatorname{Ext}_{\mathrm{FHS}_{d}}^{1}\left(\mathbb{Z}(-d), \mathrm{H}_{\sharp}^{2 d-1, d}(X)\right)
$$

which is an isomorphism by (3).

\subsection{The generalized Albanese of Faltings and Wüstholz}

Let $U$ be a smooth algebraic scheme over $\mathbb{C}$. Then it is possible to construct a smooth compactification, i.e. $\exists j: U \rightarrow X$ open embedding with $X$ proper and smooth. Moreover we can suppose that the complement $Y:=X \backslash U$ is a normal crossing divisor. ${ }^{4}$

Remark 3.6. There is a commutative diagram (See [Lek, §3])

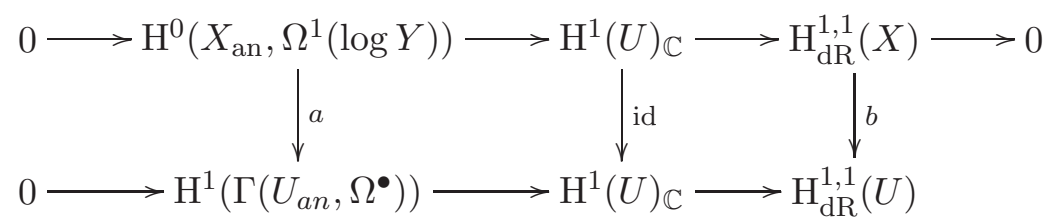

hence, by the snake lemma, Ker $b \cong$ Coker $a$. We identify these two $\mathbb{C}$-vector spaces and we denote both by $K$.

For any $Z \subset K$ sub-vector space we define the $\mathbb{C}$-linear map $\alpha_{Z}$ : $\mathrm{H}^{1}(X, \mathcal{O})^{*} \rightarrow Z^{*}$ as the dual of the canonical inclusion $Z \subset \mathrm{H}^{1}(X, \mathcal{O})$.

Definition 3.7 (The generalized Albanese of Serre). We know that

$$
\mathrm{H}^{1}(U)(1)=T_{\text {Hodge }}\left(\left[\operatorname{Div}_{Y}^{0}(X) \rightarrow \operatorname{Pic}^{0}(X)\right]\right)
$$

and that the generalized Albanese of Serre is the Cartier dual of the above 1-motive, i.e.

$$
[0 \rightarrow \operatorname{Ser}(U)]=\left[\operatorname{Div}_{Y}^{0}(X) \rightarrow \operatorname{Pic}^{0}(X)\right]^{\vee}
$$

Note that by construction $\operatorname{Ser}(U)$ is a semi-abelian group scheme corresponding to the mixed Hodge structure $\mathrm{H}^{1}(U)(1)^{\vee}:=\mathcal{H}_{\text {om }} \mathrm{MHS}\left(\mathrm{H}^{1}(U)(1), \mathbb{Z}(1)\right)$.

The universal vector extension of $\operatorname{Ser}(U)$ is

$$
0 \rightarrow \underline{\omega}_{\operatorname{Pic}^{0}(X)} \rightarrow \operatorname{Ser}(U)^{\natural} \rightarrow \operatorname{Ser}(U) \rightarrow 0
$$

this follows by the construction of $\operatorname{Ser}(U)$ as the Cartier dual of $\left[\operatorname{Div}_{Y}^{0}(X) \rightarrow\right.$ $\left.\operatorname{Pic}^{0}(X)\right]$ and [BVB06] lemma 2.2.4.

Recall that $\operatorname{Lie}\left(\operatorname{Pic}^{0}(X)\right)=\mathrm{H}^{1}(X, \mathcal{O})$, then $\underline{\omega}_{\operatorname{Pic}^{0}(X)}(\mathbb{C})=\mathrm{H}^{1}(X, \mathcal{O})^{*}$.

\footnotetext{
${ }^{4}$ It is possible to replace $\mathbb{C}$ with a field $k$ of characteristic zero. In that case we must assume that there exists a $k$ rational point in order to have $\mathrm{FW}(Z)$ defined over $\boldsymbol{k}$.
} 
Definition 3.8 (The gen. Albanese of Faltings and Wüstholz). We define an algebraic group $\mathrm{FW}(Z)$ (depending on $U$ and the choice of the vector space $Z$ ) to be the vector extension of $\operatorname{Ser}(U)$ by $Z^{*}$ defined by

$$
\alpha_{Z} \in \operatorname{Hom}_{\mathbb{C}}\left(\mathrm{H}^{1}(X, \mathcal{O})^{*}, Z^{*}\right) \cong \operatorname{Hom}_{\mathbb{C}}\left(\omega_{\operatorname{Pic}^{0}(X)}, Z^{*}\right) \cong \operatorname{Ext}^{1}\left(\operatorname{Ser}(U), Z^{*}\right)
$$

i.e. $\mathrm{FW}(Z)$ is the following push-forward

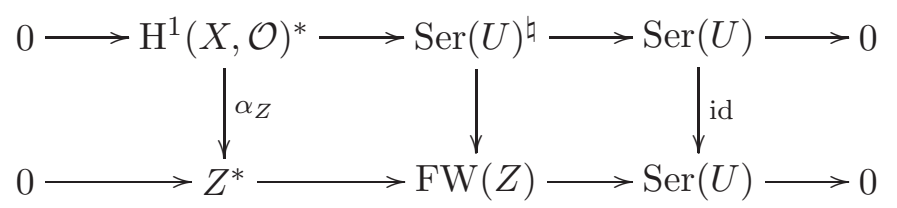

Proposition 3.9. With the above notation consider the formal Hodge structure $\left(H_{\mathrm{et}}, V\right) \in \mathrm{FHS}_{1}$ represented by

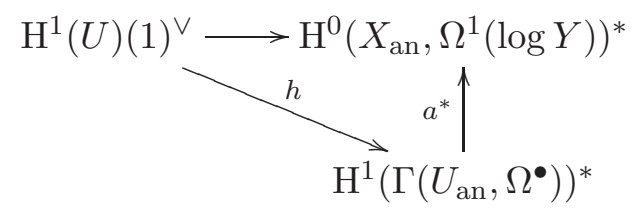

(This diagram is the dual of the left square in remark 3.6). Recall that $K=\operatorname{Ker} a$. Then

$$
\mathrm{FW}(K)_{\mathrm{an}} \cong \operatorname{Ext}_{\mathrm{FHS}_{1}}^{1}\left(\mathbb{Z}(-1),\left(H_{\mathrm{et}}, V\right)\right)
$$

Proof. It is a direct consequence of 2.10 .

\section{References}

[BS02] Spencer Bloch and Vasudevan Srinivas. Enriched Hodge structures. In Algebra, arithmetic and geometry, Part I, II (Mumbai, 2000), volume 16 of Tata Inst. Fund. Res. Stud. Math., pages 171-184. Tata Inst. Fund. Res., Bombay, 2002.

[BV07] Luca Barbieri-Viale. Formal Hodge theory. Math. Res. Lett., 14(3):385-394, 2007.

[BVB06] Luca Barbieri-Viale and Alessandra Bertapelle. Sharp de Rham realization. arXiv:math/0607115v1, 2006.

[Car87] James A. Carlson. The geometry of the extension class of a mixed Hodge structure. In Algebraic geometry, Bowdoin, 1985 (Brunswick, Maine, 1985), volume 46 of Proc. Sympos. Pure Math., pages 199-222. Amer. Math. Soc., Providence, RI, 1987. 
[Del71] Pierre Deligne. Théorie de Hodge. II. Inst. Hautes Études Sci. Publ. Math., (40):5-57, 1971.

[ESV99] Hélène Esnault, Vasudevan Srinivas, and Eckart Viehweg. The universal regular quotient of the Chow group of points on projective varieties. Invent. Math., 135(3):595-664, 1999.

[KS90] Masaki Kashiwara and Pierre Schapira. Sheaves on manifolds, volume 292 of Grundlehren der Mathematischen Wissenschaften [Fundamental Principles of Mathematical Sciences]. Springer-Verlag, Berlin, 1990. With a chapter in French by Christian Houzel.

[Lek] Silke Lekaus. On Albanese and Picard 1-motives with additive factors. (In preparation).

[Maz] Nicola Mazzari. Cohomological dimension of Laumon 1-motives up to isogenies. arXiv:0809.4926v1.

[PS08] Chris A. M. Peters and Joseph H. M. Steenbrink. Mixed Hodge structures, volume 52 of Ergebnisse der Mathematik und ihrer Grenzgebiete. 3. Folge. A Series of Modern Surveys in Mathematics [Results in Mathematics and Related Areas. 3rd Series. A Series of Modern Surveys in Mathematics]. Springer-Verlag, Berlin, 2008. 